\title{
PREVENÇÃO DE LESÕES NASAIS SECUNDÁRIAS AO USO DE PRESSÃO POSITIVA CONTÍNUA NAS VIAS AÉREAS (CPAP) EM RECÉM- NASCIDOS PREMATUROS DE EXTREMO BAIXO PESO
}

\author{
Adirléia Machado ALVES 1 \\ Eliziana Renata Souza dos SANTOS ${ }^{2}$ \\ Tathiana Ghisi de SOUZA ${ }^{3}$
}

\begin{abstract}
${ }^{1}$ Especialista em Fisioterapia Neonatal pela Universidade Estadual de Campinas - UNICAMP, a_malves@yahoo.com.br ${ }^{2}$ Especialista em Fisioterapia Neonatal pela Universidade Estadual de Campinas - UNICAMP, elizmont_23@hotmail.com

${ }^{3}$ Mestre em Saúde da Criança e do Adolescente pela Universidade Estadual de Campinas - UNICAMP, tathi_neo@yahoo.com.br
\end{abstract}

\section{Recebido em: 10/10/2013 - Aprovado em: 19/12/2013 - Disponibilizado em: 15/01/2014}

RESUMO: A pressão positiva contínua nas vias aéreas por via nasal (CPAP nasal) é um modo ventilatório comum utilizado em recém-nascidos. Sua aplicação é feita por prongas nasais que podem lesar as narinas causando desconforto e desfiguramento a longo prazo. O trauma nasal é uma complicação que surge pelo fato do prematuro apresentar a pele muito sensível e delicada, susceptível às lesões. O alívio da pressão é a chave para a cura e, principalmente, para a prevenção. Quando um trauma nasal ocorre em crianças que são dependentes desse modo de ventilação as estratégias alternativas visam proporcionar alívio e prevenir lesões. Portanto o objetivo deste estudo foi de realizar uma análise de literatura sobre as principais formas de prevenção de lesões nasais secundárias ao uso de CPAP em recém-nascidos prematuros. A pesquisa foi realizada de março a setembro de 2010, nas bases de dados da BIREME, Pubmed e MEDLINE, referentes às publicações dos últimos 30 anos, com os seguintes descritores: lesão nasal, CPAP, recémnascidos prematuros. Concluimos com este estudo que o CPAP nasal é um modo ventilatório muito utilizado em Neonatologia e passível de complicações. Atualmente, existem diversos dispositivos usados com o objetivo de reduzir a pressão da pronga na região nasal, a atenção da equipe quanto ao posicionamento da pronga nasal, seu tamanho ideal e a utilização dos dispositivos preventivos existentes é de suma importância para a redução de lesões nasais induzidas pelo uso de CPAP.

Palavras-chave: Pressão positiva contínua nas vias aéreas. Recém-nascido. Lesão nasal. Prevenção. Prematuros.

\begin{abstract}
The continuous positive airway via the nose (nasal CPAP) is a commonly used mode of ventilation in neonates. Its application is made by nasal prongs that can damage the nostrils causing discomfort and disfigurement in the long term. The nasal trauma is a complication that arises because of premature to make the skin very sensitive and delicate, prone to injury. The pressure relief is the key to healing and especially for prevention. When a nasal trauma occurs in children who are dependent on this mode of ventilation alternative strategies aim to provide relief and prevent injuries. Therefore the objective of this study was to conduct a literature review on the main ways of preventing injuries secondary to the use of nasal CPAP in preterm infants. The survey was conducted from March to September 2010 , the databases BIREME, Pubmed and Medline, referring to publications of the last 30 years, with the following descriptors : injury, nasal CPAP, premature newborns. We conclude from this study that nasal CPAP ventilation mode is widely used in neonatology and prone to complications. Currently, there are several devices used in order to reduce the pressure in the nasal prongs, the team's attention on positioning the nasal prongs, its optimal size and use of existing preventive devices is of paramount importance to the reduction of nasal lesions induced by CPAP
\end{abstract} Keys-word: Airway continue positive pressure. Newborns. Nasal injury. Prevention.Premature.

\section{I - INTRODUÇÃO}

Nos últimos 50 anos, os avanços tecnológicos ocorridos na área da saúde, em especial nas áreas perinatal e neonatal e a formação de profissionais especializados, levou a melhora da qualidade de assistência ao RN (AVERY, 1999; SEGRE, 2003).

O aumento da sobrevida de neonatos, cada vez mais prematuros, é um exemplo bastante pertinente da influência do desenvolvimento tecnológico e do aprimoramento do conhecimento científico na 
assistência neonatal (SADECK, 2004).

Uma pesquisa realizada pelo National Institute of Child Health and Human Development (NICHD) relatou que a sobrevida de RNMBP aumentou de $77 \%$ em $1987 / 1988$ para $86 \%$ em 1999/2000 (FANAROFF; HACK; WALSH, 2003; HINTZ; et al., 2005; WILSON-COSTELLO et al., 2005; HACK; FANAROF, 1990). No início de 2000, os índices de sobrevida se estabilizaram em aproximadamente $85 \%$ para RNMBP e 70\% para RNEBP (WILSONCOSTELLO et al., 2007; FANAROFF et al., 2007).

No Brasil, as taxas de sobrevida em um hospital universitário de nível terciário de atenção variavam entre 0 e $34 \%$ para prematuros com idade gestacional inferior a 26 semanas e entre 40 e $60 \%$ para os recémnascidos entre 27 e 28 semanas gestacionais, no período compreendido entre 1996 e 2000 (SADECK et al, 2001).

O suporte ventilatório não invasivo, Pressão Positiva Contínua nas Vias Aéreas (CPAP) e Ventilação por Pressão Positiva Intermitente Nasal (NIPP), tem sido usado precocemente e com maior frequência para o tratamento de doenças respiratórias em recém-nascidos prematuros.

Consequentemente, recém-nascidos de baixo peso e extremo baixo peso podem ficar no NIPP e CPAP por longos períodos de tempo (CARLISLE et al., 2009).

O CPAP é uma forma não invasiva de ventilação. Uma das complicações da aplicação do CPAP nasal é a lesão nasal, variando de edema à necrose da columela (GÜNLEMEZ et al., 2010).

Entre os diversos tipos ventilatórios, o CPAP é o que garante melhor troca gasosa e reduz o esforço respiratório. No CPAP a pressão transpulmonar positiva é continuamente aplicada nas vias aéreas durante um ciclo respiratório para evitar a eliminação completa do gás inspirado, mantendo a capacidade residual funcional, aumentando a pressão intra-alveolar e sua estabilidade, melhorando a troca gasosa e impedindo o colapso das vias aéreas durante esforço respiratório (NASCIMENTO et al., 2009). No entanto, especula-se que com lesão permanente no interior da narina, há maior risco de produção excessiva de secreções nasais, levando a apnéia obstrutiva e sequelas adversas, sendo, portanto, observado um aumento na incidência de complicações relacionadas ao uso de CPAP (YONG; CHEN; BOO, 2005).

\section{II - OBJETIVO}

Realizar uma análise de literatura sobre as formas de Prevenção de lesões nasais secundárias ao uso de CPAP em recémnascidos prematuros de muito baixo peso.

\section{III - Metodologia}

Realizou-se um levantamento bibliográfico, de março à setembro de 2010 , 
através dos sistemas nacionais (BIREME) e internacionais (PubMed) que se referem às publicações dos últimos 30 anos. Foram utilizados os seguintes descritores em português e inglês: pressão positiva contínua nas vias aéreas, causas e prevenção de lesão nasal em prematuros, utilização da pressão positiva contínua nas vias aéreas em neonatologia.

Neste estudo, utilizamos 28 artigos, onde, os critérios de inclusão foram: pesquisas publicadas dentro dos últimos 30 anos que relatassem as causas e prevenções de lesão nasal em prematuros e as características da modalidade ventilatória CPAP, bem como seus riscos em relação a lesão nasal. Os artigos que não encaixassem nesses aspectos foram excluídos do estudo.

\section{IV - REVISÃO DE LITERATURA}

\section{Recém-nascido Prematuro (RNPT)}

De acordo com a classificação da Organização Mundial de Saúde, são considerados recém-nascidos prematuros aqueles com idade gestacional inferior a 37 semanas (GOULART, 2004).

O RNPT apresenta características específicas de sua pele, tais como poucas camadas de estrato córneo, com veias visíveis e superficiais, número reduzido de fibras de fixação entre as camadas e pouco volume de fibras de colágeno e de elastina; os anexos cutâneos são imaturos e a hipoderme é atrofiada (SANTOS et al., 2007).
Diante desses fatores, é de suma importância que se tenha um cuidado especial com a pele do recém-nascido, dentro da unidade de terapia intensiva neonatal (UTIN) (ROLIM et al., 2009).

\section{Pressão Positiva Contínua das Vias Aéreas (CPAP)}

George Gregory descreveu primeiro o uso de CPAP em 1971 para o tratamento precoce da síndrome da angústia respiratória (GREGORY et al., 1971).

Em recém-nascidos, o CPAP ajuda a estabilizar a parede torácica e fornece um aumento do volume corrente durante a inspiração. O volume pulmonar pode ser preservado por redução do tempo expiratório, prevenindo o esvaziamento pulmonar completo (BUETTIKER et al., 2004).

Diversos sistemas estão disponíveis atualmente como o tubo nasofaríngeo, diferentes tipos de prongas e máscara de CPAP (BUETTIKER et al., 2004).

O objetivo do CPAP nasal é pressurizar a nasofaringe e pulmões dos recém-nascidos, no entanto um dos problemas associados a sua utilização é que a pronga nasal raramente se fixa firmemente dentro da narina. Causando vazamento de gaz e dificultando a manutenção do gradiente de pressão de dentro do nariz; o que significa que o nível do CPAP é raramente mantido na faringe (KAHN et al., 2007; DE PAOLI et al., 2005).

A melhor maneira para reduzir a perda 
de gás nasal é garantir que as prongas sejam de tamanho suficiente para caber confortavelmente nas narinas sem prejudicar o local. Uma cinta no queixo pode ser usada para reduzir o escoamento pela boca, mas na prática clínica normalmente tem pequena diferença no total escoado, particularmente porque é muito difícil manter a boca fechada e também porque a boca contribui pouco para o vazamento (DAVIS; MORLEY; OWEN, 2009).

O uso de pressão positiva contínua nasal da via aérea está se tornando cada vez mais popular como um método de sustentação respiratória no recém-nascido. Uma das complicações da aplicação de CPAP é o ferimento nasal, variando do edema à necrose, que pode ocorrer com o uso de todos os tipos dos prongas nasais (GÜNLEMEZ et al., 2010).

A pronga nasal é o dispositivo de conexão às vias aéreas mais utilizado devido à maneira simples de ofertar pressão positiva, menos invasiva, disponível em diferentes tamanhos e constituída de material leve e flexível. Apesar das vantagens, esse dispositivo pode lesar as narinas causando desconforto em longo prazo (NASCIMENTO et al., 2009).

\section{Lesões nasais associadas ao uso de CPAP}

O trauma nasal é uma complicação bem documentada do suporte respiratório não-invasivo e esta incidência tem sido relatada como tão alta quanto $40 \%$
(CARLISLE et al., 2009).

O mecanismo fundamental de ferimento nasal provocado pelo CPAP parece ser a pressão gerada na columela pelas prongas, a etiologia é semelhante aos da úlcera de pressão, as úlceras de pressão são melhores definidas como ferimento dos tecidos moles resultantes de uma pressão sobre uma proeminência óssea (THOMAS, 2001).

O aumento da pressão na columela causa diminuição da circulação do fluxo sanguíneo. Isto, subsequentemente, prejudica a perfusão tecidual e leva a isquemia com dano tecidual. Eritema persistente, lesões na pele, edema, insensibilidade e finalmente úlceras podem ocorrer. O alívio da pressão é o caminho para a cura e para a prevenção (GÜNLEMEZ et al., 2010).

Quanto ao posicionamento, uma pronga bem posicionada é aquela que não deforma a face do neonato e sua ponta não encosta no septo nasal, além de não permitir a movimentação deste dispositivo dentro das narinas (NASCIMENTO et al., 2009).

O tamanho e a fixação inadequada da pronga são fatores essenciais para efeito não benéfico e formação de lesões, principalmente em relação às prongas menores, pelo fato de as mesmas não se encaixarem perfeitamente nas narinas do neonato, acarretando fricção do dispositivo no interior das narinas, além de favorecer o escape de ar (YONG et al., 2005).

O trauma nasal significativo pode limitar o uso do CPAP nasal em alguns 
recém-nascidos prematuros que necessitam de contínuo suporte respiratório e uma maneira alternativa pode ser benéfica (SMITH et al., 2003).

Um recente estudo randomizado por Yong et al., 2005, encontrou uma alta incidência de trauma nasal devido ao CPAP e também encontrou que não há diferença significante entre prongas e máscaras. Os ferimentos nasais relatados na literatura classificam-se em rubor, eritema, crostas e escoriações. Os locais comuns para os ferimentos são a base do septo, onde encontra-se o filtro, causado pela máscara, e a interface medial do septo, causada pelas prongas. A duração do CPAP nasal é uma definição para o fator de risco de trauma nasal (SHANMUGANANDA; RAWAL, 2007).

\section{Prevenção}

$\mathrm{O}$ uso do CPAP nasal se torna grave após o uso prolongado, causando necrose das narinas e deixando a borda nasal circular. A necrose nasal que podem progredir em necrose septal, podendo ocorrer depois de apenas alguns dias do uso de CPAP nasal (ROBERTSON et al., 1996).

Quando um trauma nasal ocorre em crianças prematuras que são dependentes de CPAP, as estratégias alternativas incluem o uso de uma única pronga nasofaríngea com uma interface ou prongas nasais macias conectadas a um sistema de alta frequência (CARLISLE et al., 2009).

Para prevenir o ferimento nasal, o dispositivo não deve ser empurrado contra o septo nasal. Os ferimentos podem ocorrer dentro do nariz e no septo nasal se as prongas não forem posicionadas corretamente dentro das narinas. As erosões do septo nasal podem ser a causa de uma inexplicável septicemia em recém-nascidos em CPAP nasal. A seleção de tamanhos corretos de prongas, constante vigilância da equipe de enfermagem e atenção correta quanto ao posicionamento são necessários para prevenção de ferimentos nasais durante uso do CPAP (DAVIS; MORLEY; OWEN, 2009).

Tradicionalmente, o dispositivo estático como uma almofada de gel e superposições acolchoadas são usadas para reduzir a pressão e superfície de suporte. Um curativo de silicone pode ser usado para remanejar as úlceras de pressão. A lâmina com gel de silicone é um material macio e flexível. Isto reduz a pressão na columela, distribuindo a pressão ao redor das narinas e reduzindo a fricção entre o dispositivo e a pele (GÜNLEMEZ et al., 2010).

Em um trabalho de Günlemez et al., 2010, que utilizaram uma lâmina de silicone na superfície nasal em recém-nascidos submetidos ao CPAP nasal, encontraram que a aplicação de proteção de silicone nasal reduziu a taxa de lesões nasais e diminuiu a severidade dos ferimentos nasais como necrose da columela nasal (GÜNLEMEZ et al., 2010).

Tamanho de touca ou gorro, quando maior para a cabeça do neonato, causa 
mobilidade da tubulação, induzindo, consequentemente, a pressão das prongas no interior das narinas. É recomendável garantir toucas de tamanhos adequados que melhor se adaptem à cabeça dos neonatos (ROBERTSON et al, 1996).

No intuito de proporcionar alívio e prevenir lesões, empregam-se proteções de narinas adesivas, como esparadrapo comum, fitas hipoalergênicas e placas de hidrocolóide, utilizadas anteriormente às narinas, para evitar a fricção direta das prongas com a columela e o septo (ROBERTSON et al, 1996).

O hidrocolóide é o material mais citado, na atualidade, principalmente na literatura internacional, como medida preventiva para o aparecimento de lesões (YONG et al., 2005). Mesmo assim, lesões ainda são observadas, porém,em menor proporção. Esse fato deixa claro que questões relacionadas ao custo-benefício deveriam ser reavaliadas em centros de estudo que utilizam prongas (NASCIMENTO et al., 2009).

\section{V - DISCUSSÃO}

As lesões nasais em neonatos por uso de pressão positiva contínua nas vias aéreas com pronga é assunto pouco discutido na literatura brasileira. Contudo, as referências, principalmente as internacionais, enfatizam que complicações nasais secundárias à pronga estão presentes, principalmente, em neonatos prematuros e de baixo peso, devido à imaturidade pulmonar, necessitando, assim, de CPAP precocemente (BERNADETTE et al., 1994; MALTA; ZACONETA, 2005; YONG; CHEN; BOO, 2005; ROBERTSON et al., 1996).

Em um trabalho realizado por Nascimento et al., 2009, com o objetivo de determinar a frequência de lesão nasal em neonatos com CPAP nasal, foi observado que todos os neonatos em uso de CPAP com pronga, em um período mínimo de dois dias, apresentaram lesões. E, dos 147 neonatos avaliados, $123(83,7 \%)$ tinham idade gestacional inferior a 37 semanas.

Em 2005, Yong e colaboradores compararam o fluxo respiratório em 89 crianças com uso de máscaras nasais de silicone com o uso de prongas nasais. Não houveram diferenças significantes na incidência de trauma (29\% em máscaras versus $35 \%$ com prongas, $\mathrm{p}=0,5)$, embora estados diferentes de lesões foram observadas. Além disso, foram observados traumas nasais ocasionados tanto pela pronga nasal quanto pela máscara nasal. Yong relata que as taxas de trauma aumentaram com o tempo de utilização do CPAP.

Lesões nasais significantes pode limitar o uso de CPAP em algumas crianças prematuras que precisam de suporte respiratório contínuo. Em um relato de caso, descrito por Carlisle e colaboradores, 2009, no qual foi utilizado uma forma de CPAP oral, em virtude de uma lesão nasal importante, foi possível observar que o CPAP oral apresentou-se clinicamente mais efetivo e 
foi bem tolerado, foi ofertado por um período prolongado de tempo, permitindo a cura da mucosa nasal. Entretanto o $\mathrm{CPAP}$ oral não tem sido descrito em crianças prematuras, embora no início de 1970 as máscaras faciais eram amarradas para oferecer CPAP para a criança (SMITH et al., 2003).

Günlemez e colaboradores, 2010, avaliaram a eficácia da utilização do silicone em gel como forma de prevenção das lesões nasais pelo uso de CPAP. Dos 179 recémnascidos avaliados, $87(48,6 \%)$ receberam CPAP nasal sem a utilização do silicone em gel e $92(51,4 \%)$ foram ventilados após a aplicação do silicone em gel. Destes, 14,9\% dos recém-nascidos que não receberam a aplicação de silicone em gel apresentaram lesões nasais, ao passo que somente $4,3 \%$ daqueles que receberam o silicone em gel apresentaram lesões. Foi observado também que as crianças com lesões nasais apresentaram um tempo prolongado de uso do CPAP quando comparado com as crianças sem a lesão nasal.

Nascimento e colaboradores, em 2009 , em seu estudo, utilizaram prongas, nas quais, $19,73 \%$ eram consideradas adequadas em relação aos pacientes, ao passo que $80,24 \%$ eram consideradas inadequadas, e todas eram menores em relação ao tamanho ideal. Para os pesquisadores, a pronga considerada adequada é aquela não tão grande, chegando a estender as narinas, e nem pequena, com o espaço sobrando entre a pronga e as narinas. Neste mesmo estudo, a partir das observações feitas, supôs-se que a formação das lesões nasais tem relação com a inadequada fixação das prongas pelos profissionais de saúde. Esses introduzem as hastes das prongas totalmente na narina de modo que a ponte desse dispositivo permaneça em contato direto com a columela. Outra suposição seria o tamanho menor que o ideal, acarretando mobilidade do dispositivo dentro da narina e pinçamento do septo, conferindo o alto índice de lesões nasais decorrentes do CPAP nasal.

\section{VI - CONCLUSÃO}

O CPAP nasal é um modo ventilatório muito utilizado nas UTIN como um método de suporte ventilatório no RN. Mas ainda assim, há complicações da aplicação de CPAP como o ferimento nasal causado pela pronga.

O tamanho e a má fixação da pronga acarretam em fricção da mesma nas narinas, podendo causar um desconforto ao $\mathrm{RN}$ à longo prazo.

Atualmente, existem dispositivos, como almofada de gel e superposições acolchoadas, o uso de hidrocolóide, que tem como objetivo reduzir a pressão da pronga na região nasal.

Portanto, é de suma importância que a equipe tenha atenção quanto ao posicionamento da pronga na narina, o tamanho correto da touca ou do gorro, quanto aos dispositivos preventivos existentes, para que se consiga diminuir as incidências de lesão nasal induzida pelo CPAP dentro das UTIN, 
durante o seu uso.

\section{VII - REFERÊNCIAS}

1 - Avery, G.B. Neonatologia: perspectivas da década de 1990. In: Avery, G.B.; Fletcher, M.A.; Macdonald, M.G. Neonatologia:

fisiopatologia e tratamento. Rio de Janeiro: Medsi; 1999. p. 3-7.

2 - Bernadette C.; Loftus M.D.; Jeffrey A, Joseph H. Neonatal nasal deformities secondary to nasal continuous positive airway pressure. Laryngoscope. 1994; 104:1019-22.

3 - Buettiker, V.; Hug, M.I.; Baezinger, O.; Meyer, C.; Frey, B.. Advantages and Disadvantages of different nasal CPAP systems in newborns. Intensive Care Medicine. 2004; 30: 926-930.

4 - Carlisle, H.R.; Kamlin, C.O.F.; Owen, L.S.; Davis, P.G.; Morley, C.J. Oral continuous positive airway pressure (CPAP) following nasal injury in a preterm infant. Archive Disease Childhood Fetal and Neonatal. 2009; 95: 142-143.

5- Davis, P.G.; Morley, C.J.; Owen, L.S. Non-invasive respiratory support of preterm neonates with respiratory distress: continuous positive airway pressure and nasal intermittent positive pressure ventilation. Seminars in fetal e neonatal medicine. 2009; 14: 14-20.

6 - De Paoli, A.G.; Lau, R.; Davis, P.G.; Morley, C.J. Pharyngeal pressure in preterm infants receiving nasal continuous positive airway pressure. Archives Diseases in Childhood Fetal and Neonatal. 2005; 90: 79-81.

7 - Fanaroff, A.A.; Hack, M.; Walsh, M.C. The NICHD neonatal research network: changes in practice and outcomes during the first 15 years. Semin Perinatol. 2003; 27(4): 281-287.

8 - Fanaroff, A.A.; Stoll, B.J.; Wright, L.L.;
Carlo, W.A.; Ehrenkranz, R.A.; Stark, A.R.; et al. Trends in neonatal morbidity and mortality for very low birthweight infants. Am J Obstet Gynecol. 2007; 196(2): 147, 141-8.

9 - Goulart, A.L. Assistência ao recémnascido pré-termo. In.: Kopelman, B.I.; et al. Diagnóstico e Tratamento em

Neonatologia. São Paulo: Atheneu, 2004. p. $17-22$.

10 - Günlemez, A; Isken, T.; Gökalp, A.S.; Türker, G.; Arisoy, E.A. Effect of silicon gel sheeting in nasal injury associated with nasal CPAP in preterm infants. Indian Pediatrics. 2010; 47: 265-268.

11 - Gregory, G.A.; Kitterman, J.A.; Phibbs, R.H.; Tooley, W.H.; Hamilton, W.K.

Treatment of the idiopathic respiratorydistress syndrome with continuous positive airway pressure. The New England Journal of Medicine. 1971; 284:1333-1340.

12 - Hack, M.; Fanaroff, A.A. Outcomes of children of extremely low birthweight and gestational age in the 1990s. Semin Neonatol. 2000; 5(2): 89-106.

13 - Hintz, S.R.; Poole, W.K.; Wright, L.L.; Fanaroff, A.A.; Kendrick, D.E.; Laptooket, A.R.; et al. Changes in mortality and morbidities among infants born at less than 25 weeks during the post-surfactant era. Arch

Dis Child Fetal Neonatal. 2005; 90(2): 128133.

14 - Kahn, D.J.; Courtney, S.E.; Steele, A.M.; Habib, R.H. Unpredictability of delivered bubble nasal continuous positive airway pressure: role of bias flow magnitude and nares-prong air leaks. Pediatric Research. 2007; 62: 343-347.

15 - Malta M.V.; Zaconeta C.A.; editors. Verificação da técnica de CPAP nasal e dos parâmetros oferecidos aos recém nascidos em quatro hospitais da rede pública do Distrito Federal.[monografia na Internet]. Distrito Federal: Residência Médica em Pediatria; 2005. Disponível em: w w w · p a u 1 o m a r g 
o t to.com.br/documentos /disserta\%C3\%A7\%C3\%A3o\%20PDF.pdf

16 - Nascimento, R.M.; Ferreira, A.L.C.; Coutinho, A.C.F.P.; Veríssimo, R.C.S.S.

Frequencia de lesão nasal em recém-nascidos por uso de pressão positiva continua nas vias aéreas com pronga. Revista Latino americana de Enfermagem. 2009; 17 (4): 489-494.

17 - Robertson, N.J.; Mccarthy, L.S.; Hamilton, P.A.; Moss, A.L.H. Nasal deformities resulting from flow driver continuous positive airway pressure.

Archives Diseases in Childhood Fetal and Neonatal. 1996; 75: 209-212.

18 - Rolim, K.M.C.; Farias, C.P.X.; Marques, L.C.; et al. Atuação da enfermeira na prevenção de lesão de pele do recém nascido. Revista enfermagem. 2009; 17 (4): 544-549.

19 - Sadeck LSR. O recém-nascido pré-termo extremo e os limites de viabilidade. In:

Programa de Atualização em Neonatologia (PRORN)/Sociedade Brasileira de

Pediatria. Porto Alegre: Artmed; 2004. p. 6786.

20 - Sadeck, L.S.R.; Barros, J.C.R.; Moraes, F.M., et al. Evolução das taxas de sobrevida de RNPT nos últimos 5 anos em unidade neonatal de risco. In: 15 Congresso Brasileiro de Perinatologia: Florianópolis, SC; 2001. p. 289.

21 - Santos, R.R.R.; Cardoso, M.V.L.M.L.; Silva, G.R.F.; et al. Aplicação de manual educativo sobre a pele do recém-nascidos com estudantes de enfermagem. Revista

Eletrônica de Enfermagem: v. 9, n. 3, p. 759 $-771.2007$.

22 - Segre, C.A.M. Avanços em

neonatologia. Pediatria Moderna. 2003.

23 - Shanmugananda, K.; Rawal, J. Nasal

trauma due to nasal continuous positive airway pressure in newborns. Arch Dis Child Fetal Neonatal. 2007; 92, F 18.
24 - Smith, P.L.; O'Donnel, C.PP.; Allan, L.; et al. A physiologic comparison of nasal and oral positive airway pressure. Chest. 2003; 123: $689-694$.

25 - Thomas, D.R. Prevention and treatment of pressure ulcers: What works? What doesn't? Cleveland Clinic Journal of Medicine. 2001; 68: 704-707.

26 - Wilson-Costello D.; Friedman, H.; Minich, N.; Siner, B.; Taylor, G.; Schluchter, M.; et al. Improved survival rates with increased neurodevelopmental disability for extremely low birth weight infants in the 1990s. Pediatrics. 2005; 115(4): 997-1003.

27 - Wilson-Costello, D.; Friedman, H.; Minich, N.; Siner, B.; Taylor, G.; Schluchter, M.; et al. Improved neurodevelopmental outcomes for extremely low birth weight infants in 2000-2002. Pediatrics. 2007; 119(1): 37-45.

28 - Yong, S.C.; Chen, S..J.; Boo, N.Y. Incidence of nasal trauma associated with nasal prong versus nasal mask during continuous positive airway pressure treatment in very low birthweight infants: a randomised control study. Archives Diseases in Childhood Fetal and Neonatal. 2005; 90: 480-483. 